\title{
Structuralism and Meta-Mathematics ${ }^{1}$
}

\author{
Simon Friederich
}

\begin{abstract}
The debate on structuralism in the philosophy of mathematics has brought into focus a question about the status of meta-mathematics. It has been raised by Stewart Shapiro in (Shapiro, 2005), where he compares the ongoing discussion on structuralism in category theory to the Frege-Hilbert controversy on axiomatic systems. Shapiro outlines an answer according to which meta-mathematics is understood in structural terms and one according to which it is not. He finds both options viable and does not seem to prefer one over the other. The present paper reconsiders the nature of the formulae and symbols meta-mathematics is about and finds that, contrary to Charles Parsons' influential view, meta-mathematical objects are not "quasiconcrete". It is argued that, consequently, structuralists should extend their account of mathematics to meta-mathematics.
\end{abstract}

\section{Structuralism and the Individuation of Objects}

The main idea of mathematical structuralism is, in the words of Michael Resnik, "that in mathematics the primary subject-matter is not the individual mathematical objects but rather the structures in which they are arranged." ${ }^{2}$ The structuralist denies mathematical objects the ontological independence which is ascribed to them by traditional platonist accounts of mathematics and focuses on the relations that obtain between them. According to the structuralist account of mathematical axiom systems, Geoffrey Hellman explains, "the 'objects' involved serve only to mark 'positions' in a relational system; and the 'axioms' governing these objects are thought of not as asserting definite truths, but as defining a type of structure of mathematical interest." 3 As emphasized in (Shapiro, 2005), this point relates the structuralist doctrine to the position Hilbert defended in his famous controversy with Frege about the proper conception of axiomatic systems. As widely known, Hilbert employs the axioms in his Foundations of Geometry (Hilbert, 1959) as implicit definitions of the terms they contain. They perform some sort of normative role fixing the meanings of geometrical concepts and ought not to be read as assertions about objects with intrinsic properties specified in advance.

In Frege's view, on the other hand, it is impossible for the axioms to be used as mere implicit definitions characterizing a certain type of mathematical structure. For him, definitions and axioms play totally different roles in

\footnotetext{
${ }^{1}$ Please cite the published version: Erkenntnis, 73:67-81

${ }^{2}$ See (Resnik, 1997), p. 201

${ }^{3}$ See (Hellman, 2005), p. 537, the emphasis is Hellman's.
} 
the formulation of a mathematical theory: Systematically, definitions come first and have to fix the meanings of all concepts the mathematical theory under consideration makes use of. It is only after the definitions have unambiguously fixed the meanings of mathematical concepts that the axioms can express genuine truths, something Frege sees as their intrinsic task. He therefore argues that when the axioms are formulated the meanings of all concepts involved are "assumed to be known in advance." 4

Since structuralism is a conception of mathematical objects, we might like to transform the Fregean doctrine into a statement concerning not only mathematical concepts and their meanings but mathematical objects themselves. This can be done by translating it into a statement concerning the criteria of identity for mathematical objects. If the meanings of mathematical concepts are, as Frege demands, "assumed to be known in advance", mathematical objects have to be thought of as being individuated independently of and prior to their appearance in an axiomatic system. From the structuralist point of view, however, the reverse is true. The structuralist, who regards the axioms as implicitly defining a type of mathematical structure, sees mathematical objects as characterized exhaustively by their relations to other objects within the same structure. Therefore, on the structuralist account of mathematical axiom systems, mathematical objects are individuated only within the system itself and reference to them is always in the "context of some background structure." 5

In terms of criteria of identity the structuralist account of mathematical objects can be formulated as the following structuralist thesis (ST):

(ST) Criteria of identity for the objects of mathematics must be specified exclusively in terms of the relations obtaining between them.

(ST) provides the basis for the considerations about meta-mathematics presented in the following sections. I shall not speak about what generally speaks in favor of structuralism in the philosophy of mathematics nor shall I discuss the most important objections that have been levelled against it. Rather I shall be concerned with structuralism and meta-mathematics, that part of mathematics about which it is commonly said that it studies mathematics itself. My aim is to show that, contrary to what is claimed by some influential authors ${ }^{6}$, structuralism has no particular problems with metamathematics so that structuralists should have no qualms extending their

\footnotetext{
${ }^{4}$ For the German original see (Frege, 1976), an English translation can be found in (Frege, 1980). It should be emphasized that Frege's views on arithmetic and geometry are strongly different. Frege, while he aimed at a logicist reduction of arithmetic, insisted on a substantial role of intuition in geometry. Both his accounts on arithmetic and geometry, however, are in conflict with the Hilbertian approach according to which the axioms are used as implicitly defining the concepts they contain.

${ }^{5}$ This formulation is due to Parsons, see (Parsons, 1990) p. 303.

${ }^{6}$ See, for example, the claims brought forward in (Shapiro, 2005) and the final section of (Parsons, 1990).
} 
account to meta-mathematics.

An issue which has been hotly debated more recently is whether structuralism is a plausible position with respect to mathematical theories the objects of which are arranged in structures that admit of non-trivial automorphisms (such as the complex numbers under complex conjugation). Claims equivalent or similar to (ST) have been criticized by different authors (Burgess, 1999), (Keraenen, 2001), (MacBride, 2005), (MacBride, 2006), (Button, 2006) on the charge that according to (ST) there could be no numerical diversity of structurally indistinguishable objects. Since the criticism of these authors is motivated by metaphysical considerations about individuation, the most promising strategy for defending structuralism is to turn the attention away from metaphysics to mathematical practice. This strategy is pursued in (Ketland, 2006), (Ladyman, 2005), (Leitgeb and Ladyman, 2008), and (Shapiro, 2008). Similarly, the present paper does not operate on the basis of metaphysical principles about individuation but looks at the criteria of identity in the context of our actual practices. ${ }^{7}$

The rest of this paper is organized as follows: In the next section it is discussed why meta-mathematics seemingly poses a serious problem for any structuralist interpretation. The apparent problem is that its objects seem to be what Charles Parsons has called quasi-concrete objects and that quasiconcrete are individuated not merely according to the relations obtaining among them. This challenge for structuralism is taken up in Sections 3 and 4 where it is argued that, in fact, the objects of meta-mathematics are not quasi-concrete, but that the standard application of meta-mathematics is to the study of linguistic systems whose elements are quasi-concrete. It is argued that therefore structuralism faces no particular problems with respect to meta-mathematics. Finally, in Section 5 the considerations of the foregoing sections are summarized and a few words are said about their more general relevance for structuralists accounts.

\footnotetext{
${ }^{7}$ There already exists a study that focuses on criteria of identity for meta-mathematical objects, namely (Mühlhölzer, forthcoming), and the ideas put forward there have helped to formulate the account of meta-mathematics presented here. Mühlhölzer explores the resources of the later Wittgenstein's philosophy of mathematics to accommodate the existence of meta-mathematics where Wittgenstein seems to run into trouble. There are parallels between Mühlhölzer's analysis and the one presented here for the case of structuralism. As an example, the structuralist with respect to meta-mathematics holds that the objects of meta-mathematics are individuated only according to their relations to other such objects (in a way to be clarified in Section 4), while similarly they are "not given in advance" with respect to the meta-mathematical "calculus", or so Mühlhölzer's later Wittgenstein holds. Mühlhölzer argues that the idea of studying criteria of identity can be found in the work of Wittgenstein, see (Wittgenstein, 1958) p. 62.
} 


\section{The Problem of Giving a Structuralist Account of Meta-Mathematics}

Meta-mathematics is commonly characterized as that portion of mathematics in which the properties of mathematical theories themselves are investigated. For Hilbert meta-mathematics was essentially proof theory, the syntactical investigation of proofs carried out in formal axiomatic systems, yielding results about, e. g., the consistency of the systems. However, many important proofs and results which are normally regarded as part of metamathematics are based on concepts and methods belonging to other parts of mathematics such as model theory (e.g. the completeness theorem) or recursion theory (results in reverse mathematics, for instance). Having a notion of meta-mathematics which extends beyond mere proof theory seems therefore advisable. Nevertheless, for the purposes of the present paper a precise definition of meta-mathematics is not needed, for it suffices to focus on those aspects of meta-mathematics which seem to be the most problematic from a structuralist point of view. These, as will become clear in a minute, are the syntactical, "proof-theoretical", aspects.

Structuralism, according to its formulation in the structuralist thesis (ST) stated above, is wrong with respect to theories the objects of which are individuated independently of the relations obtaining among them. But this is what seems to be case with regard to the objects of proof theory- the symbols and formulae mathematical proofs in formal systems are "concatenations" of. It seems natural to think of mathematical symbols and formulae as linguistic objects and hence as objects of the kind Charles Parsons has dubbed "quasi-concrete". ${ }^{8}$ Quasi-concrete objects are objects which are not themselves concrete but have concrete instantiations and are thus directly linked to objects that are properly concrete. A letter type which corresponds to some concrete letter token (in form of an inscription on paper, say) is an example of a quasi-concrete object. As Parsons makes plausible, quasi-concrete objects are individuated independently of and prior to our making true statements about them in meta-mathematical contexts. Quoting Parsons:

In the statement of the [structuralist] view using the metalinguistic conception of structure, we appeal to linguistic objects such as predicates and functors. These are quasi-concrete objects, and so long as they are viewed in this way the structuralist view will not hold for them. The relation of linguistic types to their tokens (and in general of quasi-concrete objects to their concrete 'representations') is not an external one... ((Parsons, 1990) p. 337)

\footnotetext{
${ }^{8}$ This terminology frequently appears in (Parsons, 1990) as well as in his recent book (Parsons, 2008), but his idea of the preeminent importance of quasi-concrete objects can already be found in his now classic Mathematical Intuition (Parsons, 1980).
} 
Parsons' claim that the relation between type and token is "not an external one" can be translated into the language of individuating conditions by saying that whether some type is or can be instantiated by this or that concrete token provides a criterion of identity for the type. The symbol type, in other words, is individuated by reference to the tokens which count as its instantiations, and whether some token counts as an instantiation of the type depends, of course, on whether we actually treat it as such. Criteria of identity for symbol types, in short, are not purely relational, they are not specified exclusively in terms of relations among such types. In order to save the possibility of structuralism in meta-mathematics it is therefore necessary to show that meta-mathematics is not about quasi-concrete objects at all.

In a first and very crude attempt to defend structuralism about metamathematics the structuralist might try to show that meta-mathematical objects are not quasi-concrete by questioning the very soundness and legitimacy of the notion of quasi-concreteness, for instance by arguing that quasi-concrete objects, if they existed, would necessarily be unintelligible. No such attempt, however, seems promising to me: There exists an established and well-functioning practice of recognizing different symbol tokens as instantiations of the same (or different) symbol type, which means that there is an established practice of individuating symbol types in terms of what counts as their instantiations. Little children are able to learn what letters, words and numerals are, and they learn how the same word can be present in different inscriptions or utterings. So, what they learn to individuate are the types of the symbols, not only the tokens. Even if the perception of symbols were confined to the perception of tokens, contradicting Parsons' account of quasi-concrete objects, the individuation of symbol types seems to be something very basic and utterly unproblematic. Linguistic objects, even the types, are individuated not according to their mutual relations but according to widely recognized standards governing which tokens count as instantiations of the same type. If meta-mathematics really is about objects of this kind, structuralism with respect to meta-mathematics is undermined once and for all.

The structuralist might be tempted simply to insist on a re-interpretation of meta-mathematics along structuralist lines. This strategy is anticipated by Parsons who concedes that it is possible to interpret meta-mathematics in such a way. However, as Parsons argues, this move merely leads to a displacement of the problems the structuralist faces with respect to metamathematics:

It will be objected that any mathematical theory, that of linguistic objects included, can be interpreted as talking about objects for which the structuralist view holds. With regard to mathematical structuralism based on the metalinguistic conception of structure, the proposal is that syntax be viewed in this way, with 
the notions of string and concatenation, and perhaps others, as basic relations...

The philosophical gain [this move] achieves, however, is only apparent... The more concrete domains, often of quasi-concrete objects, still play an ineliminable role in the explanation and motivation of mathematical concepts and theories. In particular, this is true of any mathematical treatment of formalized or naturalized languages. Thus if the structuralist view of mathematical objects is taken to mean that all mathematical objects are only structurally determined, it has to rest on legislation about what counts as a mathematical object. The explanatory and justificatory role of more concrete models implies, in my view, that it is not the right legislation even for the interpretation of mathematics. ((Parsons, 1990) pp. 337-38.)

Parsons' argument seems to be that even though meta-mathematical language can in principle be interpreted along structuralist lines reasoning about quasi-concrete objects occuring in meta-mathematics remains to be accommodated. According to Parsons, the mere possibility of reinterpreting meta-mathematical proofs and theorems in a structuralist manner "afterwards", so to speak, does not suffice in order to establish a structuralist account of them because, on the original reading, our theorems about them were no less mathematical. The fact that we can construct an interpretation of meta-mathematical language which makes it accord with structuralist principles is indeed not very surprising. Parsons, I think, is right in claiming that this is not of much help to the structuralist. The problem with metamathematics is that, taken at face value and in its usual interpretation, it is a perfectly respectable part of mathematics and nevertheless seems to be in conflict with structuralism.

A variant of Parsons' objection to structuralism about meta-mathematics just cited applies to the attempt of establishing structuralism about metamathematics by first arithmetizing the language of meta-mathematics ${ }^{9}$ and subsequently endorsing structuralism about arithmetic and the arithmetized theory. This move is not a promising one, too, for although arithmetization is standard and central to some of the most ingenious proof ideas in metamathematics (including, of course, Gödel's proof of his first incompleteness theorem, see (Gödel, 1931)) it is not what makes meta-mathematical proofs meta-mathematical.

What has to be shown by anyone who wants to establish structuralism in meta-mathematics is that meta-mathematical reasoning, even in the first place, is not and has never been about linguistic objects for which Parsons' account of quasi-concreteness holds. This is the aim of the following section. In Section 4 it will then be proposed that the relation of meta-mathematics

\footnotetext{
${ }^{9}$ See (Gödel, 1931), (Feferman, 1960).
} 
to quasi-concrete objects is not one of aboutness but one of application. The apparent problem for structuralism about meta-mathematics is thereby solved.

\section{Meta-Mathematics, Symbols and "Letters"}

In this section I present the main argument for the claim that the objects of meta-mathematics which seemingly pose problems for structuralismthe symbols, formulae and proofs studied in proof theory - are not quasiconcrete and therefore not problematic from a structuralist point of view. The argument is based on a terminological distinction between types of letters and numerals on the one hand and objects which are the elements of formal languages on the other. It is argued that while the first are indeed quasi-concrete only the latter are the objects of meta-mathematics. This opens the door for structuralism about meta-mathematics.

Pursuing the meta-theory of some mathematical axiom system, say Peano arithmetic, we assume that the vocabulary of the system is given by some language $L$, a class of symbols. For Peano arithmetic, these symbols are usually represented by numerals and letters such as " 0 ", " $S$ ", or "+". Together with these symbols, the language $L$ normally contains a couple of logical constants. A formula of Peano arithmetic is any syntactically wellformed string - concatenation - of these symbols. The meta-theory of Peano arithmetic is "about" these symbols in the sense that it analyzes which sequences of them can be built according to the axioms of Peano arithmetic and the rules of logic. Since the aim of the present section is to show that structuralism may be extended to meta-mathematics it is necessary to make it plausible that these symbols are individuated only according to their relations among another.

Let us have a closer look at the nature of the elements of the formal language $L$. To do so, it is useful to consider the possibility of pursuing the metatheory of Peano arithmetic with the axioms formulated in terms of different "letters". What I have in mind is a rather simple point which starts from the observation that the linguistic objects corresponding to elements of the language of Peano arithmetic need not be those we normally decide to use: One could, of course, use the Greek letter " $\Omega$ " instead of the numeral " 0 ", the punctuation mark "' " instead of the Latin letter " $S$ ", and so on for all the other symbols in $L$, without ceasing to study the meta-theory of Peano arithmetic. It is tempting to say that one is free to choose arbitrary symbols to state the axioms of Peano arithmetic. The problem with putting the matter this way is that one may want to reserve the notion of a symbol to refer to the elements of the formal language $L$. Describing the replacement of " $S$ " by " " as employing a different symbol in the sense of formulating Peano arithmetic within another language $L^{\prime}$ instead of $L$ would be quite 
misleading. Given this ambiguity in the meaning of "symbol", I shall refer to the ordinary letters, numerals, punctuation marks like " $S$ ", " $\Omega$ ", "0" or " 1 ", which occur in numerous contexts besides the mathematical one, simply as "letters". This allows us to distinguish them from the more formal objects which are the elements of the formal language $L$.

Obviously, letters and elements of formal languages like $L$ are in a way linked or brought into contact with each other in the meta-mathematical analysis of mathematical theories. A letter is somehow "attached" to the element of the formal language when we do meta-mathematics, e.g. the letter " $S$ " to the formal object associated to the successor function. I will refer to this kind of "attachment" of a letter to an object of a formal language as a representation of the formal object by the letter. This notion of "representation" should not be confounded with that of a symbol type's being "represented" by some concrete symbol token. To denote this relation the word "instantiate" is employed in the present paper. According to this use of "representation", any letter may represent any element of any formal language, and the "letters" involved here are just those types some of which, for example, form the every-day alphabet. The letter " $S$ ", which represents the formal object associated to the successor function, is just the " $S$ " little children get to know in primary school and learn to identify as a component of numerous different words. One can think of the letter " $S$ " as a type of inscription or as a complex linguistic entity associated to some type of inscription as well as to certain phonemes. Where and how letters such as " $S$ " occur in language is something open to the analysis of linguistic study. Although the individuation of letters may be complicated and difficult to describe, the nature of letters lies open to view insofar as their individuation proceeds according to universally recognized every-day standards. These standards, which may be difficult to account for in detail, allow us to decide whether some given token is an instantiation of this or that type.

The nature of formal symbols, the elements of languages like $L$, is utterly different. Let us now turn to their criteria of identity and compare them to those for letter types. Letter types, I argued, are individuated according to which tokens count as instantiations of them. With respect to the individuation of formal symbols an analogous guess may be tried: Are they individuated according to which letters are admitted as acceptable representations of them?

Clearly no. Formal symbols can be represented by arbitrary letters. There are, for example, no limits with regard to possible representations of the formal symbol associated to the successor function. It is even thinkable that we let it be represented by different letters at the same time or allow that representations of formal symbols by letters may overlap (which means using the same letter for different symbols), and there is nothing wrong with that as long as we manage not to get confused about which formula is which. 
But what then is the principle of individuation for formal symbols?

Take, as an example, the formula " $S S O+S 0=S 0+S S 0$ ", which is a chain of elements of $L$ corresponding to a provable statement in PA. It cannot, as we have seen, be individuated by reference to any sequence of ordinary language letters such as the "letters" "S", "0", "+" and "=", for it can equally well be represented by sequences of letters which look completely different. Statements concerning which representation we employ do not deliver criteria of identity for the formal symbols and formulae treated in meta-mathematics. Therefore, other properties of these objects have to be found by reference to which criteria of identity for meta-mathematical objects can indeed be stated.

Fortunately such properties do exist. Consider, for example, the property of being derivable in Peano arithmetic or the property of being a sentence derived by using the definition of "+" or, even more simple, the property of having the formal symbol " $S$ " at its first and second, "0" at its third, "+" at its fourth place and so forth. These properties, on the one hand, uniquely characterize the formula in question and thus provide definite criteria of identity. On the other hand, they are completely independent of possible representations of the formula by sequences of letters. The structuralist will be happy to present these properties as those according to which our formula can be individuated because an immediate observation about them is that they are all relational.

The structuralist, we see, is in a good position to argue that the elements of $L$ are individuated only within meta-mathematics itself and that statements which provide criteria of identity for them are relational. The sequence of formal symbols " $S S 0$ ", for example, is individuated as just that sequence to which the sequence " $+S 0=S 0+S S 0$ " can be attached yielding their concatenation " $S S 0+S 0=S 0+S S 0$ ", a provable proposition of Peano arithmetic. ${ }^{10}$ Between some formula and a certain formal symbol there may obtain the relation of containment or, more precisely, the relation of containment at the $n$-th place. Between some set of formulae and another formula there may obtain the relation of the latter being deducible from the former. The set of the axioms of Peano arithmetic (hopefully) has the property of being consistent, which means that the sentence "SO=0" does not stand to them in the relation of derivability. The structuralist will regard the net of relations like containment between formulae and symbols as the framework in which questions about their identity make sense and can be settled.

Parsons, in his abovecited remark on potential structuralist readings of meta-mathematics, conceded that a structural interpretation of those re-

\footnotetext{
${ }^{10}$ The notion of concatenation as applied to the objects of a formal language can be spelled out formally in an axiomatic way. This has been done in (Grzegorczyk, 2005) obtaining, as a result, the Gödel-Church theorem of the undecidability of logic the proof of which is usually based on arithmetization.
} 
lations is possible, but he rejected it on the grounds that quasi-concrete objects still "play an ineliminable role in the explanation and motivation of mathematical concepts and theories". Quasi-concrete objects such as letters are of course needed to formulate any mathematical theory whatsoever, meta-mathematical theories included. But they are not the objects of these theories, the structuralist should argue. As we have just seen, a structural reading of meta-mathematics comes in naturally when we try to come to terms with the nature of the objects meta-mathematics really is about. It is not contrived and artificially implemented in order to save the structuralist doctrine in the face of hopeless difficulties, but has emerged from considerations on the principles governing the individuation of objects in meta-mathematics.

Let us now turn to the relation of "representation" between formal symbols and ordinary linguistic objects or "letters".

\section{Meta-mathematics Applied}

In the last section I have contrasted the objects belonging to formal languages as they are treated in meta-mathematics to the letters representing them. Meta-mathematics, I have argued, is only about the former and not about the latter, which may be characterized as, in Parsons' terminology, quasi-concrete. However, the quasi-concrete objects, the letters, somehow also play a role in meta-mathematics, if only due to their being "represented" by the purely formal objects. The structuralist, I suggest, should characterize the nature of this relation between the two different kinds of objects as a form of application of some part of mathematics to some realm of non-mathematical objects. Meta-mathematics, the structuralist might argue, can be described as being applied to studying the properties of systems of letters as used in mathematics.

The nature of this application of meta-mathematics to systems of linguistic objects can be sketched by comparing it to the application of mathematical geometry - analytic geometry, for instance - to physical space. The structuralist may claim that in this application the points of physical space can be said to concretely "represent" the points of analytic geometry in much the same manner as letters can be said to "represent" the formal symbols in meta-mathematics. The application of analytic geometry to physical space is of enormous help to physicists in order to predict and explain a virtually unlimited variety of observable phenomena connected to physical space. Similarly, the structuralist might argue, meta-mathematics, in its application to systems of linguistic objects, sheds some predictive and explanatory light on some of the facts observed in mathematical practice. A consistency proof for some formal axiomatic system, for instance, allows us to predict that whithin a system of linguistic objects representing those of the formal 
system no sequence of letters expressing a contradiction will ever be obtained. In case we had been wondering why no one has ever been able to come up with a contradiction in that system so far, this fact is thereby nicely understood. Meta-mathematics thus importantly contributes to our predictive and explanatory resources with respect to the facts of mathematical life. This partly justifies the way it is usually characterized, namely as that part of mathematics which is concerned with the investigation of mathematics itself. In the context of the structuralist picture of meta-mathematics proposed here, however, this investigation does not concern the subject matter of meta-mathematics itself but rather forms the, so to speak, "canonical" application of meta-mathematics.

It is characteristic of structuralism that it always looks least plausible, at least at first glance, with respect to those mathematical disciplines which are practiced in direct orientation to one specific application. In such a case every step of mathematical reasoning will always be seen in the light that it throws onto the domain of objects the mathematical theory in question is applied to. The distinction between the mathematical theory and its application constantly threatens to get out of sight (or not to be recognized in the first place). This was once the case with Euclidean geometry and still is the case with regard to meta-mathematics. Euclidean geometry for a long time was considered the mathematical theory of the concrete points and lines in physical space. Only with Hilbert's axiomatization it became clear how the structural aspects of Euclidean geometry - the genuinely mathematical ones, the structuralist would say - could be separated from the theory's application to physical space. The same development made it clear that Euclidean geometry, all by itself, is not any more about physical space than about any other system of objects for which we can find an interpretation that makes its axioms true.

Similarly, meta-mathematical theories are usually developed with one specific application in mind, the application to mathematics itself and concrete mathematical practice. This application is an application to a domain of non-mathematical objects inasmuch as it involves representations of formal objects by (sequences of) letters. Linguistic objects are, of course, needed to formulate mathematical sentences in the first place. But this renders meta-mathematics no more about letters or other linguistic objects than mathematical geometry is about real physical space.

The idea of describing the link between meta-mathematics and ordinary "object-level" mathematics not as meta-mathematics being about the latter but somehow applied to it is certainly not new. However, the only proposal I know of which develops a picture of meta-mathematics as in an important sense applied as part of a structuralist account of meta-mathematics is by Stewart Shapiro. Since this proposal interestingly differs from the one given here I briefly discuss it.

Shapiro outlines his proposal for conceiving of meta-mathematics as ap- 
plied to other parts of mathematics in his introductory "Thinking about mathematics" (Shapiro, 2001) where he discusses the deductivist's attitude towards meta-mathematics. To the deductivist, who may be afraid of running into the same or analogous troubles with respect to meta-mathematics as the structuralist, Shapiro proposes the following route:

$[\mathrm{M}]$ eta-mathematics can be (and subsequently was) formalized. To be consistent, our deductivist should propose that the 'mathematics' in meta-mathematics is just the derivation of consequences from the axioms of this meta-mathematics, with these axioms regarded as meaningless. The 'application' of metamathematics to formal languages and deductive systems is irrelevant to its essence as a branch of mathematics. Just as arithmetic can be applied to counting, meta-mathematics can be applied to deductive systems. ((Shapiro, 2001) pp. 153-54)

In contrast to the deductivist, who defends a version of formalism, the structuralist does not hold that either the axioms nor any other mathematical propositions are in fact meaningless. All she contends is that they define a certain (type of) structure. Modulo this refinement, however, Shapiro's proposal may be applied to structuralism no less than to deductivism.

Shapiro's proposal, although it conceives of meta-mathematics as in a sense applied to mathematical language, would not, at least not as it stands, fit well with the route to structuralism in meta-mathematics proposed here. The deductivist, as instructed by Shapiro, holds that meta-mathematics is applied to the study of formal languages and formal deductive systems. Given the argument presented before, however, meta-mathematics is not applied but rather straightforwardly about the elements of formal languages in just the same way as Peano arithmetic is about numbers. The fact that, according to the account proposed here, meta-mathematics is about merely formal objects and not about objects that are quasi-concrete is just what renders structuralism about meta-mathematics a viable option. The notion of an application of meta-mathematics becomes relevant only when we consider the relation of meta-mathematics to such non-formal objects as "letters" (and numerals etc.) of our ordinary language which are indeed quasi-concrete and of which it has been said that they may represent the formal objects.

In the more recent paper (Shapiro, 2005) mentioned in the abstract Shapiro extends his earlier proposal and develops it as an option specifically for those who aim at a structuralist-not deductivist-reading of meta-mathematics. In his outline of how the structuralist interpretation of mathematics could be extended to meta-mathematics the notion of an application continues to play a vital role. Shapiro argues that part of the talk involving meta-mathematical notions like satisfiability and categoricity cannot be given a structuralist interpretation, but he proposes to regard this 
piece of reasoning not as talk within meta-mathematics, but as an application of meta-mathematics: "[A]ssertory statements about interpretations, deductions, relative consistency, and the like, are an application of the background meta-theory, perhaps the standard application." ${ }^{11}$ In the context of this passage "assertory statements about interpretations, deductions, relative consistency, and the like" are statements for which Shapiro sees no perspective of a structuralist reading. According to the option for structuralism in meta-mathematics developed by him, these sentences are located outside mathematics. They are conceived of as "assertory philosophy" 12 and are thus seen as an extra-mathematical application of meta-mathematical theory, an application, i.e., to philosophy.

In this respect the option outlined by Shapiro again is markedly different from the proposal developed here. If the argument given above is correct, the structuralist can simply deny that there are, as Shapiro calls it, "statements about interpretations, deductions, relative consistency, and the like" which do not admit of a structuralist reading. The structuralist need not exclude sentences like "Peano arithmetic is consistent", involving the meta-mathematical notion of consistency, from the realm of mathematics declaring them to be extra-mathematical applications of meta-mathematics in order to motivate structuralism about meta-mathematics. With respect to the sentence "Peano arithmetic is consistent" the structuralist may argue that, as an object of meta-mathematical inquiry, Peano arithmetic is defined by reference to its language and axioms the structurally determined identity of which has been motivated above. It inherits its purely relationally given identity from them. Describing a sentence like "Peano arithmetic is consistent" as an application of meta-mathematics to assertory philosophy seems quite unnatural, for it is a statement having a (meta-) mathematical proof (the Gentzen proof) and therefore ought to be located within the boundaries of mathematics. In the route to structuralism about meta-mathematics suggested here, the application of meta-mathematics only begins when we conclude, from the Gentzen consistency result, say, that no one will ever derive a contradiction in Peano arithmetic. Arguably, this way of demarcating mathematics from non-mathematics is the more natural one.

\section{Summary and Concluding Remarks}

Let me first review the main thoughts presented in this paper:

The core idea of structuralism in the philosophy of mathematics is that mathematical objects are essentially positions in structures and, as such, individuated only according to their relations among another. My aim has been to show that, contrary to how things appear at first glance, the struc-

\footnotetext{
${ }^{11}$ See (Shapiro, 2005), p. 75.

${ }^{12}$ See (Shapiro, 2005), p. 75, further below.
} 
turalist is in a good position to argue that also in meta-mathematics the objects are individuated only by reference to other members of the structures they belong to. Pace Parsons, the objects treated in proof-theory are not quasi-concrete objects and pose no specific problems for the structuralist. Quasi-concreteness applies only to linguistic objects proper, the symbol types or "letters", of which it has been said that they "represent" the objects meta-mathematics really is about.

The argument given is not aimed at showing that structuralism about meta-mathematics is correct in all and each respect, for there is a number of challenges to structuralism in the philosophy of mathematics in general which have not been addressed. All that should have been made plausible is that meta-mathematics forms no special problem for structuralism and can be given a structuralist interpretation as non-artificially as any other part of mathematics, for example arithmetic. The precise form in which the considerations presented here become relevant for structuralism depends on the variety of structuralism one prefers and seeks to apply to metamathematics. I have implicitly assumed the structuralism to be of the noneliminativist ("realist") kind and have not made an attempt to eliminate reference to the objects of meta-mathematics altogether.

However, the main lesson from the present investigation can be incorporated into an eliminative structuralist account as well. The main difference between eliminative and non-eliminative structuralist understandings of meta-mathematics concerns the status of the structurally determined symbols and formulae. The eliminativist cannot recognize them as respectable objects in their own right. Instead, he will have to interpret the metamathematical theory as relating more directly to ordinary linguistic objects or "letters" which, as I have argued, merely represent the formal mathematical symbols proper. The most promising version of eliminative structuralism in the philosophy of mathematics today is probably Geoffrey Hellman's modal structuralism. If the line of thought proposed here is taken over into that account, meta-mathematics has to be thought of as saying something about possible linguistic objects and (possible) configurations thereof or, if radical nominalism is pursued and even quasi-concrete objects are shunned, about possible sign tokens and their configurations. Talk of possible configurations of linguistic objects is still mathematical as long as the modality in question is conceived of as modality in a specifically mathematical sense. ${ }^{13}$ The question of where the line between meta-mathematics and its application has to be drawn in such an eliminativist approach is somewhat subtle. It is closely connected to the general issue of distinguishing between different kinds of modality - in this case mathematical and physical - which by far exceeds the range of what can be discussed in the present context.

\footnotetext{
${ }^{13}$ See (Hellman, 1989), e. g. p. 15.
} 
It has been argued by Hellman ${ }^{14}$ that any structuralist account of mathematics, for the sake of ontological clarity, ought to be formulated within some background framework of statements which are not themselves interpreted structuralistically. In particular, so Hellman complains ${ }^{15}$, questions of mathematical existence have not been properly addressed by any version of structuralism in category theory that has been proposed so far. ${ }^{16}$ According to Hellman, the structuralist, in order to make her commitments in ontology and modality as explicit as possible, should specify a framework of assertions which are not themselves to be read along structuralist lines and in which these commitments are clearly laid out.

The result of the present paper - that structuralism about meta-mathematics is a viable option - may be taken to indicate that Hellman need not be followed here: As long as a structuralist account of meta-mathematics interpreting it as consisting of statements about purely relationally defined objects seems unimaginable, the natural way of specifying a framework in Hellman's sense is simply by identifying it with meta-mathematics. Different versions of structuralism are then discriminable according to what kinds of meta-mathematical reasoning they employ and admit, whether, for example, they assume primitive modality, accept excluded middle etc. But as soon as meta-mathematics has also been given a structuralist reading in a natural way the alleged need of an non-structural framework for stating structuralist accounts of mathematics appears much less compelling. This may be interpreted as encouraging anti-foundationalist varieties of structuralism such as the form of categorical structuralism proposed in (Awodey, 2004). Versions of structuralism along these lines, applying the principles of structuralism to what others conceive of as a framework for formulating their structuralist accounts, are certainly the most "structuralist" of all.

\section{Acknowledgments}

I would like to thank Wilfried Keller and Felix Mühlhölzer for stimulating discussions and suggestions of how to improve the paper. Furthermore, I am grateful to Stewart Shapiro and an anonymous referee of Erkenntnis for many helpful comments on an earlier version.

\section{References}

Awodey, S. (1996). Structure in mathematics and logic: A categorical perspective, Philosophia Mathematica (3), 4, 209-237.

\footnotetext{
${ }^{14}$ See (Hellman, 2003), (Hellman, forthcoming).

${ }^{15}$ See (Hellman, 2003), especially p. 9, and (Hellman, 2005).

${ }^{16}$ The most important contributions to structuralism in category theory include (McLarty, 1993), (Awodey, 1996), (McLarty, 2004), (Awodey, 2004).
} 
Awodey, S. (2004). Does category theory provide a framework for mathematical structuralism?, Philosophia Mathematica (3), 12, 54-64.

Burgess, J. (1999). Book Review: Stewart Shapiro. Philosophy of Mathematics: Structure and Ontology, Notre Dame J. Formal Logic, 40, 2, 283-291.

Button, T. (2006). Realist structuralism's identity crisis: A hybrid solution, Analysis, 66, 216-222.

Feferman, S. (1960). Arithmetization of metamathematics in a general setting, Fundamenta Mathematicae, 49, 35-92.

Frege, G. (1976). Wissenschaftlicher Briefwechsel, ed. by Gabriel, G., Hermes, H., Kambartel, F., and Thiel, C., (Hamburg: Felix Meiner).

Frege, G. (1980). Philosophical and mathematical correspondence, transl. by Kaal, H., ed. by McGuiness, B., (Oxford: Basil Blackwell).

Gödel, K. (1931). Über formal unentscheidbare Sätze der Principia Mathematica und verwandter Systeme, I. Monatshefte Math. Phys., 38, 173198.

Grzegorczyk, A. (2005). Undecidability without arithmetization, Studia Logica, 79, 163-230.

Hellman, G. (1989). Mathematics without Numbers, (Oxford: Oxford University Press).

Hellman, G. (2003). Does category theory provide a framework for mathematical structuralism?, Philosophia Mathematica (3), 11, 129-157.

Hellman, G.. Structuralism. (In: Shapiro, S. (2005). The Oxford Handbook of the Philosophy of Mathematics and Logic (pp. 536-562). Oxford: Oxford University Press.)

Hellman, G. (forthcoming). What is categorical structuralism?, available online at http://www.tc.umn.edu/ hellm001/.

Hilbert, D. (1899). Grundlagen der Geometrie, (Leipzig: Teubner), trans. by Townsend, E. (1959) as Foundations of Geometry, (La Salle, Illinois: Open Court).

Keränen, J. (2001). The identity problem for realist structuralism, Philosophia Mathematica (3), 9, 308-330.

Ketland, J. (2006). Structuralism and the identity of indiscernibles, Analysis, $66,303-315$. 
Ladyman, J. (2005). Mathematical structuralism and the identity of indiscernibles, Analysis, 65, 218-221.

Leitgeb, H. and Ladyman, J. (2008). Criteria of identity and structuralist ontology, Philosophia Mathematica (3), 16, 388-396.

MacBride, F. (2005), Structuralism reconsidered. (In: Shapiro, S. (2005). The Oxford Handbook of the Philosophy of Mathematics and Logic (pp. 563-589). Oxford: Oxford University Press.)

MacBride, F. (2006). What constitutes the numerical diversity of mathematical objects?, Analysis 66, 63-69.

McLarty, C. (1993). Numbers can be just what they have to, Noûs, 27, 487-498.

McLarty, C. (2004). Exploring categorical structuralism, Philosophia Mathematica (3), 12, 37-53.

Mühlhölzer, F., Wittgenstein and Metamathematics, (forthcoming in: Stekeler-Weithofer, P. (Ed.), Wittgenstein, Philosophie und Wissenschaften, Hamburg: Felix Meiner).

Parsons, Ch. (1979-80). Mathematical intuition, Proceedings of the Aristotelian Society, 80, 145-168.

Parsons, Ch. (1990). The structuralist view of mathematical objects, Synthese, 84, 303-346.

Parsons, Ch. (2008). Mathematical Thought and its Objects, (NewYork: Cambridge University Press).

Resnik, M.D. (1997). Mathematics as a Science of Patterns, (Oxford: Oxford University Press).

Shapiro, S. (2001), Thinking about Mathematics: The Philosophy of Mathematics, (Oxford: Oxford University Press).

Shapiro, S. (2005). Categories, structures, and the Frege-Hilbert controversy: The status of meta-mathematics, Philosophia Mathematica (3), 13, 6177.

Shapiro, S. (2008). Identity, indiscernibility and ante rem structuralism: The tale of $i$ and $-i$, Philosophia Mathematica (3), 16, 285-309.

Wittgenstein, L. (1958). The Blue and Brown Books, (Oxford: Blackwell). 